\title{
Atom-molecule coherence for ultralong-range Rydberg dimers
}

\author{
B. Butscher, J. Nipper, J. B. Balewski, L. Kukota, V. Bendkowsky, R. Löw and T. Pfau *
}

\begin{abstract}
An important goal in quantum chemistry is the coherent control of reversible reactions between pure initial and final states of individual constituents. Recent examples of coherent control of such chemical reactions include the photoassociation of ultracold ground-state dimer molecules ${ }^{1,2}$ and of Feshbach molecules in highly excited vibrational states ${ }^{3}$. Here, we extend coherent control of such reactions to the photoassociation of dimer states in highly excited electronic states. We demonstrate coherent transfer of initially free pairs of rubidium ground-state atoms to ultralong-range Rydberg molecules consisting of a highly excited Rydberg atom and a ground-state atom $^{4,5}$. The coherent evolution of the molecular system is monitored in echo and Ramsey-pulse sequences by measuring the timescales for the energy-conserving dephasing rate, $T_{2}$, and for non-energy-conserving decay processes, $T_{1}$. These experiments demonstrate an atom-molecule interferometer with Rydberg states.
\end{abstract}

Ultralong-range Rydberg molecules are based on an exotic binding mechanism based on attractive electron-atom scattering. Since their first experimental observation ${ }^{5}$, spectroscopic studies of these unusual molecular states have unveiled another new binding mechanism for ultralong-range Rydberg molecules based on quantum reflection ${ }^{6}$. However, the investigation of the dynamical properties of the ultralong-range Rydberg molecules demands experimental methods going beyond continuous-wave spectroscopy. In the following we demonstrate that our narrow-band laser set-up can coherently drive a system consisting of two rubidium ground-state atoms between the unbound $5 s-5 s$ pair state and the bound ${ }^{3} \Sigma(5 s-35 s)$ molecular ground state by means of a rotary echo-type technique. We model the experimental results with a two-level optical Bloch calculation. The found dephasing and decoherence properties of the system are convincingly reproduced in a Ramsey-type experiment, that is set up to realize an atommolecule interferometer.

We create the ultralong-range Rydberg molecules in the ${ }^{3} \Sigma(5 s-35 s)(v=0)$ vibrational ground state from a sample of approximately $2 \times 10^{6}{ }^{87} \mathrm{Rb}$ atoms at a temperature of $3 \mu \mathrm{K}$ in the ground state $5 s_{1 / 2}\left(F=2 ; m_{F}=2\right)$ by means of a twophoton transition. The two lasers at $780 \mathrm{~nm}$ and $480 \mathrm{~nm}$ are detuned by $\approx 400 \mathrm{MHz}$ from the intermediate $5 p_{3 / 2}$ state (see Methods). A schematic diagram of the levels and the excitation lasers is given in Fig. 1a.

Recently, Rabi oscillations of single Rydberg atoms were observed over several microseconds $s^{7,8}$. In contrast, previous coherence measurements in many-body ultracold Rydberg systems did not allow observation of coherence times of more than a few hundred nanoseconds owing to laser linewidths of $\gtrsim 1 \mathrm{MHz}$ (refs 9-11). However, for an atomic sample at a temperature of $3 \mu \mathrm{K}$, we expect a Doppler broadening of only $125 \mathrm{kHz}$ full-width at half-maximum as the dominant line-broadening mechanism. Therefore, we have
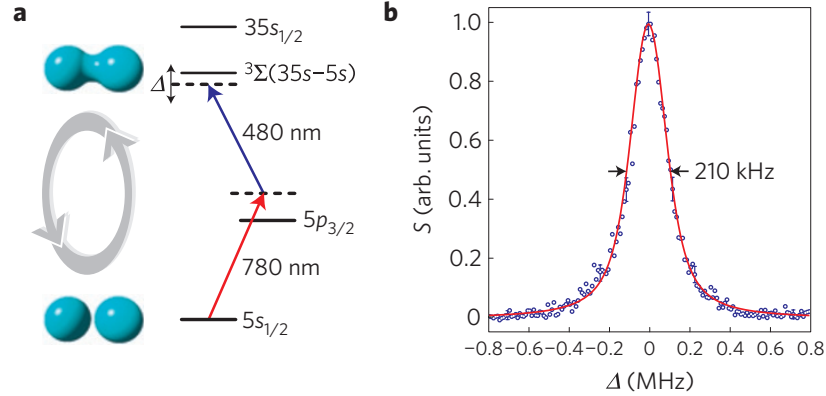

Figure 1 | Excitation of ultralong-range Rydberg molecules. a, Scheme of the photoassociation of ultralong-range Rydberg molecules. The red laser is detuned from the intermediate $p$ level by about $400 \mathrm{MHz}$. The blue laser frequency is tuned on two-photon resonance to the ${ }^{3} \Sigma(5 s-35 s)(v=0)$ state of the molecule, separated by its binding energy $E_{\mathrm{B}}=-22.8 \mathrm{MHz}$ from the atomic 35 s Rydberg state. $\mathbf{b}$, Spectrum of the molecular state ${ }^{3} \Sigma(5 s-35 s)(v=0)$. For one atomic sample, we excite the cloud for $20 \mu \mathrm{s}$ at each frequency and average the scan over 11 samples. The exemplary error bars show the standard deviation. The red line is a Voigt line profile for a Doppler temperature of $3 \mu \mathrm{K}$ and a Lorentzian linewidth of $142 \mathrm{kHz}$.

set up a narrow-band laser excitation system with linewidth below the Doppler limit, which enables us to test for much longer coherence times. To determine the linewidth of our combined laser system, we employ a delayed self-heterodyne interferometer ${ }^{12}$ using a 10-km-long fibre as delay line. For both excitation lasers we independently obtain linewidths of less than $20 \mathrm{kHz}$. Taking also into account the effects of subsequent optical elements, that is, acousto-optic modulators and optical fibres, we estimate a total linewidth of $\Delta v<100 \mathrm{kHz}$ for our excitation system.

An exemplary high-resolution spectrum of the vibrational ground state of the ultralong-range Rydberg molecules is shown in Fig. 1b. The full-width at half-maximum is $210 \mathrm{kHz}$. As our measurement of the temperature of $3 \mu \mathrm{K}$ is accurate within $5 \%$, we can fit a Voigt profile with fixed Gaussian lineshape to our data. This gives us a full-width at half-maximum linewidth of $142 \mathrm{kHz}$ for the Lorentzian part, which is larger than our laser linewidth, and thus is not limited by technical constraints. The Voigt line profile with the corresponding Gaussian and Lorentzian parts is plotted in Fig. $1 \mathrm{~b}$ and reproduces the observed lineshape well, indicating that the entire inhomogeneous linewidth is only due to Doppler broadening.

As a proof of coherent photoassociation, we apply a rotary echo sequence $^{13,14}$ that we have already successfully used to investigate the $43 \mathrm{~s}$ state of rubidium atoms ${ }^{10}$. A square laser pulse of fixed length $\tau$ and Rabi frequency $\Omega$ on resonance $\Delta=0$ excites pairs of suitably spaced rubidium ground-state atoms to the molecular ground state. After a time $\tau_{\mathrm{p}}<\tau$, we invert the phase of the driving 
laser field $\Omega \rightarrow-\Omega$ with an acousto-optic modulator (see Fig. 2). In a fully coherent system, this is equivalent to a time reversal in the evolution of the dynamics.

For each atomic sample, we vary the time of inversion from $\tau_{\mathrm{p}}=0$ to $\tau_{\mathrm{p}}=\tau$ and observe the number of Rydberg molecules created, $S$. We repeat this sequence for different pulse lengths $\tau$ and average over five atomic samples for each value. Exemplary results of these measurements are shown in Fig. 2a. For short pulses of $1.6 \mu \mathrm{s}$, we can see that the ultralong-range Rydberg molecules are almost completely transferred back into the unbound ground state if the phase is inverted at $\tau_{\mathrm{p}}=\tau / 2$, which is the expected behaviour for a coherent excitation. Even for long pulses of several microseconds, half of the molecules can be detached again, clearly demonstrating the coherent control of ultralong-range Rydberg molecules on the timescale of microseconds.

To compare the results of different pulse lengths, we fit a parabola to the experimental values and calculate the visibility of the echo signal for each pulse duration defined by

$$
v=\frac{\max (S)-\min (S)}{\max (S)+\min (S)}
$$

with $\max (S)$ and $\min (S)$ being the maximal and minimal values of the fitted Rydberg signal. The experimentally found visibility $v$ for different pulse durations $\tau$ is shown in Fig. $2 b$.

To model the dynamics of the photoassociation process, we assume that the two-level system consisting of the unbound ground pair state and the molecular state can be described by optical Bloch equations ${ }^{15,16}$,

$$
\begin{gathered}
\dot{u}=-\Delta v-\frac{u}{T_{2}} \\
\dot{v}=\Delta u+\Omega w-\frac{v}{T_{2}} \\
\dot{w}=-\Omega v-\frac{w+1}{T_{1}}
\end{gathered}
$$

where $\Delta$ is the detuning, $\Omega$ the two-photon Rabi frequency and $T_{1}$ and $T_{2}$ are known as longitudinal and transverse relaxation times in NMR. In quantum optics, the relaxation time $T_{1}$ is the excited-state lifetime and describes the population decaying to the ground state by means of spontaneous emission. $T_{2}$ is the coherence lifetime and accounts for all losses of coherence, for example radiation loss and laser linewidth. The population of the molecular state is given by $S=(w+1) / 2$.

To obtain the relaxation time $T_{1}$ and the coherence time $T_{2}$ from the experimental data, we follow Torrey's method ${ }^{17}$ and solve equation (1) analytically for $\Delta=0$. Then we use a least-square algorithm to find the $\left(T_{1}, T_{2}\right)$ pair fitting best to the experimental data. For the measurements in Fig. 2 we find for the lifetime of the excited state $T_{1}=6.4(9) \mu \mathrm{s}$ and a coherence lifetime of $T_{2}=1.5(2) \mu \mathrm{s}$. Note that the $T_{2}$ lifetime is shorter than the upper limit given by our laser linewidth and the loss of coherence due to $T_{1}$ decay. The obtained theoretical curves are shown in grey in Fig. $2 \mathrm{a}$ and red in Fig. 2b. The good agreement between experimental results and our calculations clearly demonstrate the validity of this model. In particular, the value of $T_{1}$ can be considered as a precise spectroscopic determination of the lifetime of the ultralongrange Rydberg molecules, which is significantly shorter than measured previously ${ }^{5}$. This discrepancy may be explained if we take into account that previously a delayed field-ionization experiment was used to determine the lifetime of the Rydberg population. As blackbody radiation induces decays to closely adjacent Rydberg states which are field ionized as well ${ }^{18,19}$,

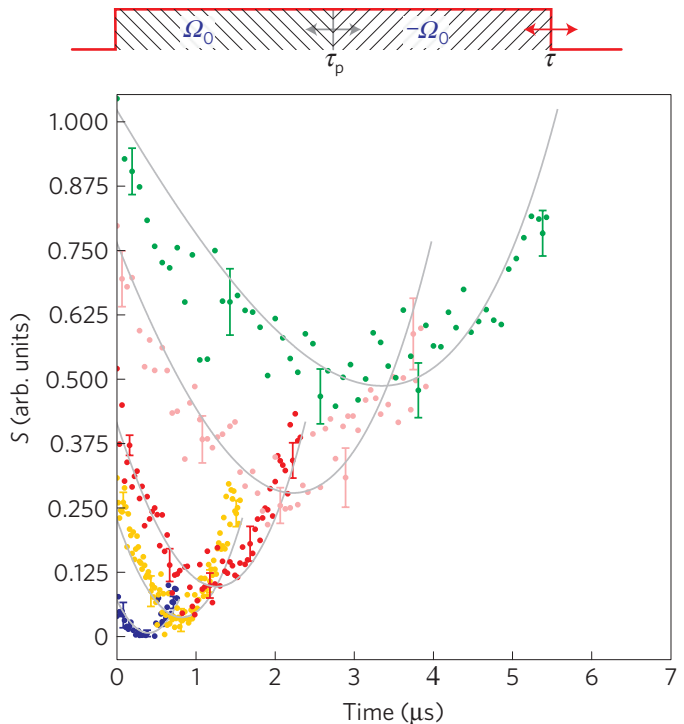

b

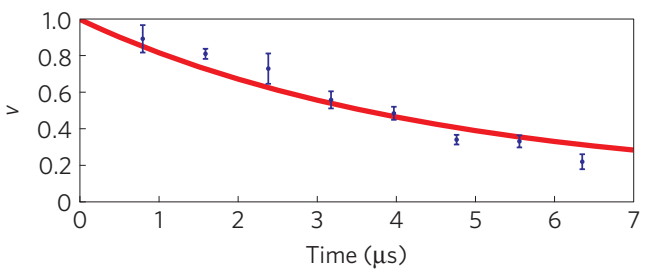

Figure $\mathbf{2}$ | Rotary echo experiments of ultralong-range Rydberg molecules. For a square pulse of fixed duration $\tau$, the time of phase inversion was varied in one atomic sample from $\tau_{\mathrm{p}}=0$ to $\tau_{\mathrm{p}}=\tau$. $\mathbf{a}$, Echo curves for pulse durations of $0.8 \mu \mathrm{s}$ (blue), 1.6 $\mathrm{\mu s}$ (yellow), $2.4 \mu \mathrm{s}$ (red), $3.2 \mu \mathrm{s}$ (pink) and $4.8 \mu \mathrm{s}$ (green). The grey lines are the echo curves we obtain from our calculations and an asymmetry becomes visible if the pulse duration becomes comparable to the lifetime $T_{1} . \mathbf{b}$, Visibility $v$ of the echo signal as a function of the duration of the excitation pulse. The red line is the result from our theoretical model. All points are averaged over five atomic samples each and the error bars represent the standard deviation.

the lifetime of the total Rydberg population in ref. 5 also accounts for the lifetime of the adjacent states and thus can be longer than the lifetime of the bound-pair state we measure in the present experiment.

To challenge the validity of our study of the dynamic properties of the photoassociation of the molecules, we extend the rotary echo experiments to finite detunings of the excitation laser. According to the principle that spectroscopically obtained information is in general equivalent to information obtained in the time domain, the coherence of the molecular association is also visible in spectroscopy of the molecular state.

Therefore, we repeat the echo experiments with a fixed pulse length of $\tau=1.6 \mu \mathrm{s}$ and vary the time of inversion in one sample from $\tau_{\mathrm{p}}=0$ to $\tau_{\mathrm{p}}=\tau$. The detuning to the molecular resonance is varied by $50 \mathrm{kHz}$ from sample to sample within $\Delta= \pm 1.4 \mathrm{MHz}$. The resulting colour-coded $\Delta-\tau_{\mathrm{p}}$ map is shown in Fig. 3a. Since an analytic solution of equation (1) is only feasible for $\Delta=0$, we numerically integrated the optical Bloch equations with the lifetimes we obtained previously. The results are shown in Fig. 3b. The agreement between theory and experiment and the consistency of the input parameters $T_{1}$ and $T_{2}$ from two distinct experiments clearly demonstrate the coherent nature of the photoassociation and that the echo sequences constitute a suitable tool to explore its properties.

Since the coherence lifetime is on the order of microseconds, it is experimentally feasible to set up Ramsey experiments 


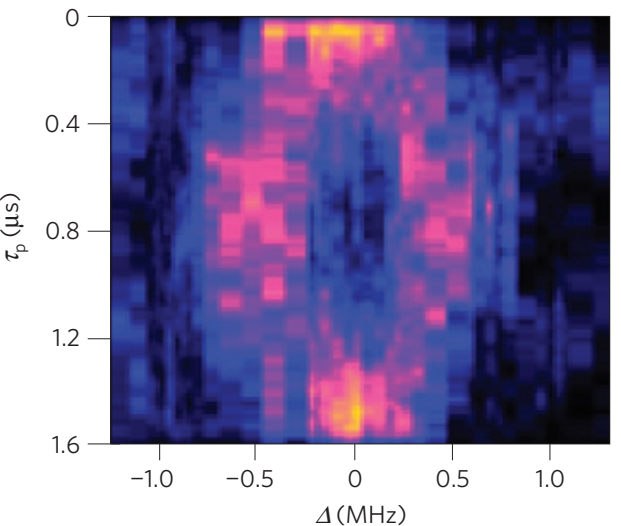

b

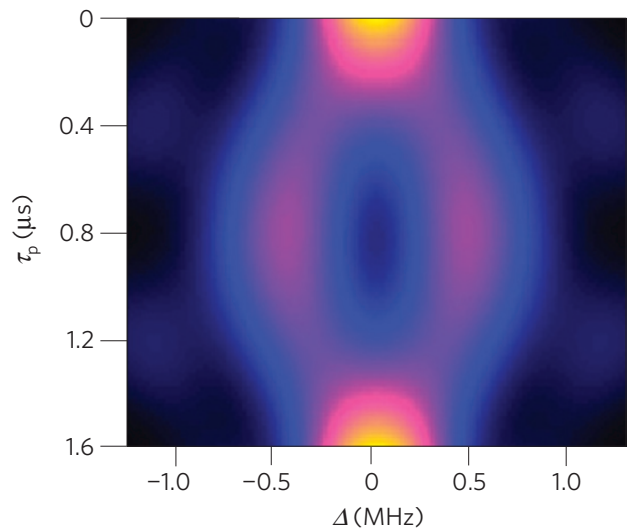

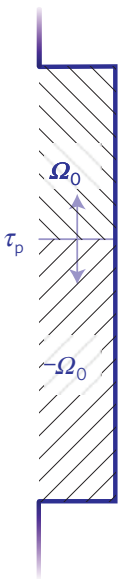

Figure $\mathbf{3}$ | Rotary echo experiments with variable detuning. a,b, For a fixed detuning $\Delta$ from the molecular resonance, we take one echo curve with an excitation pulse of duration $\tau=1.6 \mu$ s and vary the time of phase inversion from $\tau_{\mathrm{p}}=0 \mu \mathrm{s}$ to $\tau_{\mathrm{p}}=\tau$. The Rydberg signals we obtain in experiment (a) and theory (b) are given as colour-coded plots normalized to the maximum Rydberg signal.
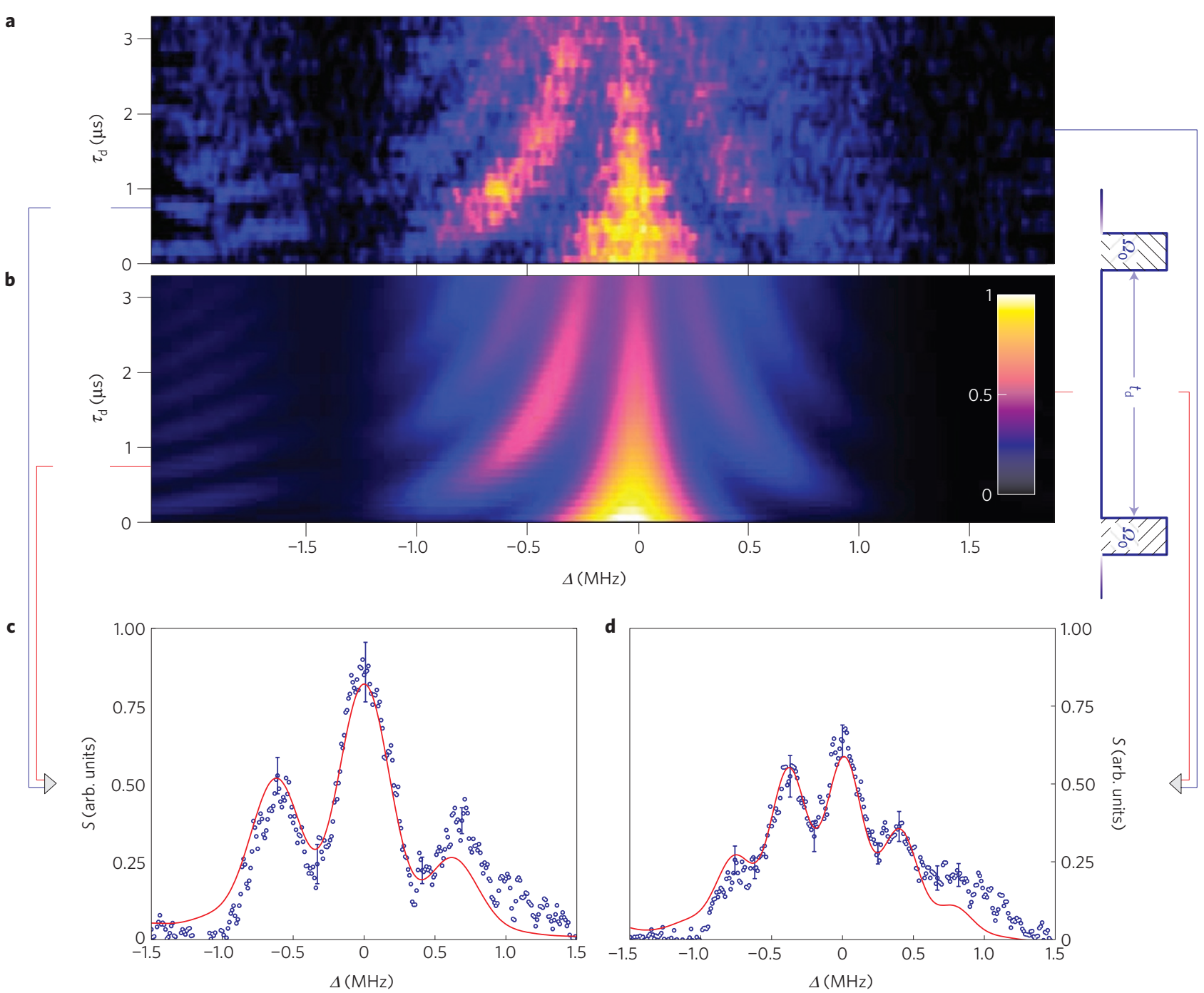

Figure 4 | Ramsey experiment for ultralong-range Rydberg molecules. We excite our atomic sample with two 0.75 - $\mu$ s-long laser pulses separated by a wait time $\tau_{\mathrm{d}}$. We scan our laser in one sample from $\Delta \approx-2.0 \mathrm{MHz}$ to $\Delta \approx 2.0 \mathrm{MHz}$ over the molecular resonance. This scan is repeated for wait times from $\tau_{\mathrm{d}}=0 \mu \mathrm{s}$ to $\tau_{\mathrm{d}}=3.3 \mu \mathrm{s}$. a, Colour-coded normalized experimental Rydberg signal. The decreasing signal from left to right is due to a slow depletion of ground-state atoms. We account for this in our calculation by an exponential decay. $\mathbf{b}$, Results of our two-level model calculation with $T_{1}=6.4 \mu$ s and $T_{2}=1.5 \mu$ s. c,d, Cuts along the $\Delta$-axis for fixed wait times of $\tau_{\mathrm{d}}=0.7 \mu \mathrm{s}$ and $\tau_{\mathrm{d}}=1.7 \mu$ s with experimental (blue circle s) and theoretical (red curve) results. Points in $\mathbf{c}$ and $\mathbf{d}$ are averaged over eight atomic samples each and the error bars represent the standard deviation. 
where one excitation pulse is followed by a second, identical, pulse after a variable delay time ${ }^{20}$. There are several advantages of Ramsey-type experiments over echo experiments. In echo experiments, the exciting laser drives the system during the whole experimental sequence, causing decoherence and atom loss due to scattering from the intermediate $p$ state. Ramsey's method demands only two short pulses at the beginning and at the end of the sequence without any laser field during the delay time and thus decoherence and atom losses are reduced in this scheme. Moreover, Ramsey experiments enable us to precisely detect external manipulation of the system during the delay time, which enables us to consider them as an interferometer between the unbound ground state and ultralong-range molecular state. Compared with ground-state atoms, Rydberg atoms have an exaggerated sensitivity to external perturbations, for example electric fields or the presence of other Rydberg atoms nearby, owing to their huge interaction strength. For a superposition between a pair of ground-state atoms and an ultralong-range molecule this means that the relative phase of the molecular state can exhibit enormous shifts. A subsequent recombination pulse maps the relative phase into a shifted population of ultralong-range Rydberg molecules that can be measured easily, thus enabling a precise measurement of the phase shift.

To extend our scheme to a Ramsey method, we use a pulse of fixed duration $\tau=0.75 \mu$ s to create a superposition of the ground state and the ultralong-range molecule state followed by a variable delay time $\tau_{\mathrm{d}}$ to enable the relative phase to evolve and a second pulse of duration $\tau=0.75 \mu$ s to map the relative phase back to populations of the ground and molecular states. For each cloud, we keep the delay time fixed and scan our laser by $4 \mathrm{MHz}$ across the molecular resonance. Then we repeat this experiment in a newly prepared cloud for delay times $0 \mu \mathrm{s} \leq \tau_{\mathrm{d}} \leq 3.2 \mu$ s. A Ramsey fringe pattern in the horizontal $\Delta$-direction is clearly visible in the experimental data in Fig. 4a. The vanishing signal for increasing detuning is caused by a slow depletion of trapped ground-state atoms and does not affect the coherent evolution. The width of the envelope is given by the duration of the pulse as $1 / \tau$ and the frequency spacing of the Ramsey fringes by the delay time as $1 / 2 \tau_{\mathrm{d}}$, as can be seen from the scan along the frequency axis in Fig. $4 \mathrm{c}$ and d. Employing the previously found values for $T_{1}$ and $T_{2}$ in the calculation for the Ramsey experiment and accounting for the depletion of pair states by means of an exponential decay yields the results depicted in Fig. $4 \mathrm{~b}$. In Fig. $4 \mathrm{c}$,d we show scans along the $\Delta$-axis for delay times $\tau_{\mathrm{d}}=0.7 \mu \mathrm{s}$ and $\tau_{\mathrm{d}}=1.7 \mu \mathrm{s}$ as blue circles with the results of the calculation overlaid as red curves. This conventionally used display of the results allows for both an easier comparison to other Ramsey-type experiments and judgement of the excellent agreement between experiment and theory. Aside from the coherence of the photoassociation, the occurrence of Ramsey fringes confirms the realization of an atom-molecule interferometer. The relative phase shift between ground state and molecular state for given delay time $\tau_{\mathrm{d}}$ and detuning $\Delta$ in Fig. $4 \mathrm{a}$ is given by $\delta \varphi=2 \pi \Delta \tau_{\mathrm{d}}$ in the case of no external perturbation. It can be seen in Fig. 4 that for a fixed delay time $\tau_{\mathrm{d}}$ the maxima of the population of the ultralong-range-molecule state are separated in detuning $\Delta$ by $1 / 2 \tau_{\mathrm{d}}$. This confirms that we obtain maxima in population for phase shifts of $\delta \varphi=n \pi$ and minima for $\delta \varphi=(2 n+1) / 2 \pi$ and demonstrates that we have successfully mapped the relative phase shift on the molecular population.

The prototype of an interferometer between ultralong-range Rydberg molecules and ground-state atoms that we have realized will enable us to study the evolution of the phase of this exotic molecular state in a precise and well controllable way. If, for example, the molecular ground state is coupled to a vibrationally excited state during the delay time, time-resolved information about the vibration can be deduced from the Rydberg population after the second pulse. However, interferometric methods may also serve to understand the considerably reduced lifetime of the molecular state compared to the bare atomic state because this demands a time-resolved analysis of the dynamics. The experimental observation of ultralong-range Rydberg molecules spotlighted existing ideas such as Rydberg Borromean trimer states $^{21}$ and initiated new concepts such as the coherent control of molecular orientation states ${ }^{22}$. The methods we have presented in this letter will help to pave the way towards the realization of these ideas and to gain a deeper understanding of this new and exotic binding mechanism. Extending the scheme of Rydberg dressing of ground-state atoms that enables us to control the two-body interaction in a Bose-Einstein condensate ${ }^{23}$ to ultralongrange Rydberg molecules, we expect that a coherent admixture of interacting molecule-molecule states to the Bose-Einstein mean field will enable us to design four-body interactions in an ultracold quantum gas.

\section{Methods}

To excite the ultralong-range Rydberg molecules, we prepare a sample of approximately $2 \times 10^{6}{ }^{87} \mathrm{Rb}$ atoms at a temperature of $3 \mu \mathrm{K}$ in the ground state $5 s_{1 / 2}$ $\left(F=2 ; m_{F}=2\right)$. In all experiments, we address the ${ }^{3} \Sigma(5 s-35 s)(v=0)$ vibrational ground state of the ultralong-range Rydberg molecules by means of a two-photon transition. The two lasers are detuned by $\approx 400 \mathrm{MHz}$ from the intermediate $5 p_{3 / 2}$ state. The laser light is generated by two master-slave diode-laser set-ups at $780 \mathrm{~nm}$ and at $961 \mathrm{~nm}$ being locked to a passively stabilized cavity. The $961 \mathrm{~nm}$ laser is frequency doubled to $480 \mathrm{~nm}$. The $480-\mathrm{nm}$ laser has a power of $50 \mathrm{~mW}$ and is focused down to a waist of $165 \mu \mathrm{m}$. The power of the 780 -nm laser varies between $2 \mu \mathrm{W}$ for the spectrum, $10 \mu \mathrm{W}$ in the rotary echo sequences and $100 \mu \mathrm{W}$ for the Ramsey sequence and has a waist of $3 \mathrm{~mm}$. We calculate two-photon Rabi frequencies of $30 \mathrm{kHz}$ for the spectrum, $50 \mathrm{kHz}$ for the echo experiment and $180 \mathrm{kHz}$ in the Ramsey sequence.

Received 3 August 2010; accepted 29 September 2010; published online 21 November 2010

\section{References}

1. Ospelkaus, S. et al. Quantum-state controlled chemical reactions of ultracold potassium-rubidium molecules. Science 327, 853-857 (2010).

2. Deiglmayr, J. et al. Formation of ultracold polar molecules in the rovibrational ground state. Phys. Rev. Lett. 101, 133004 (2008).

3. Donley, E. A., Claussen, N. R., Thompson, S. T. \& Wieman, C. E. Atom-molecule coherence in a Bose-Einstein condensate. Nature 417 529-533 (2002).

4. Greene, C. H., Dickinson, A. S. \& Sadeghpour, H. R. Creation of polar and nonpolar ultra-long-range Rydberg molecules. Phys. Rev. Lett. 85, 2458-2461 (2000).

5. Bendkowsky, V. et al. Observation of ultralong-range Rydberg molecules. Nature 458, 1005-1008 (2009).

6. Bendkowsky, V. et al. Rydberg trimers and excited dimers bound by internal quantum reflection. Phys. Rev. Lett. 105, 163201 (2010).

7. Urban, E. et al. Observation of Rydberg blockade between two atoms. Nature Phys. 5, 110-114 (2009).

8. Gaëtan, A. et al. Observation of collective excitation of two individual atoms in the Rydberg blockade regime. Nature Phys. 5, 115-118 (2009).

9. Heidemann, R. et al. Evidence for coherent collective Rydberg excitation in the strong blockade regime. Phys. Rev. Lett. 99, 163601 (2007).

10. Raitzsch, U. et al. Echo experiments in a strongly interacting Rydberg gas. Phys. Rev. Lett. 100, 013002 (2008).

11. Younge, K. C. \& Raithel, G. Rotary echo tests of coherence in Rydberg-atom excitation. New J. Phys. 11, 043006 (2009).

12. Okoshi, T., Kikuchi, K. \& Nakayama, A. Novel method for high resolution measurement of laser output spectrum. Electron. Lett. 16, 630-631 (1980).

13. Wong, N. C., Kano, S. S. \& Brewer, R. G. Optical rotary echoes. Phys. Rev. A 21, 260-267 (1980).

14. Solomon, I. Rotary spin echoes. Phys. Rev. Lett. 2, 301-302 (1959).

15. Bloch, F. Nuclear induction. Phys. Rev. 70, 460-474 (1946).

16. Allen, L. \& Eberly, J. Optical Resonance and Two-level Atoms (Dover Publications, 1987).

17. Torrey, H. C. Transient nutations in nuclear magnetic resonance. Phys. Rev. 76, 1059-1068 (1949). 
18. Beterov, I. I., Ryabtsev, I. I., Tretyakov, D. B. \& Entin, V. M. Quasiclassical calculations of blackbody-radiation-induced depopulation rates and effective lifetimes of Rydberg nS, $\mathrm{nP}$, and $\mathrm{nD}$ alkali-metal atoms with $n \leq 80$. Phys. Rev. A 79, 052504 (2009).

19. Nascimento, V. A., Caliri, L. L., de Oliveira, A. L., Bagnato, V. S. \& Marcassa, L. G. Measurement of the lifetimes of S and D states below $n=31$ using cold Rydberg gas. Phys. Rev. A 74, 054501 (2006).

20. Ramsey, N. F. A new molecular beam resonance method. Phys. Rev. 76, 996-996 (1949).

21. Liu, I. C. H., Stanojevic, J. \& Rost, J. M. Ultra-long-range Rydberg trimers with a repulsive two-body interaction. Phys. Rev. Lett. 102, 173001 (2009).

22. Rittenhouse, S. T. \& Sadeghpour, H. R. Ultracold giant polyatomic Rydberg molecules: Coherent control of molecular orientation. Phys. Rev. Lett. 104, 243002 (2010)

23. Honer, J., Weimer, H., Pfau, T. \& Büchler, H. P. Collective many-body interaction in Rydberg dressed atoms. Phys. Rev. Lett. 105, 160404 (2010).

\section{Acknowledgements}

We thank H. P. Büchler, J. Thywissen, J-M. Rost and T. Pohl for discussions. B. Butscher thanks the Carl Zeiss Stiftung for financial support. This work is funded by the Deutsche Forschungsgemeinschaft (DFG) within SFB/TRR21 and the project PF 381/4-1.

\section{Author contributions}

The experiment was conceived by B.B., J.N. and T.P. and carried out by B.B., J.N., J.B.B., L.K. and V.B.; data analysis was accomplished by B.B. and J.N.; theory, numerical algorithms and implementations are by B.B.; B.B. wrote the manuscript; V.B., R.L. and T.P. contributed to the manuscript.

\section{Additional information}

The authors declare no competing financial interests. Reprints and permissions information is available online at http://npg.nature.com/reprintsandpermissions. Correspondence and requests for materials should be addressed to T.P. 